\title{
The Utilization of Formal Medical Services for Young Children
}

The lack of impact of medical professionals in social networks

Finn Rasmussen

Department of Paediatrics, Children's University Hospital, Sweden

\begin{abstract}
The objective of this study was to examine the impact of medical professionals in social networks on the use of formal medical care for young children's minor illnesses. 795 Swedish mothers, with only one child 5-15 months of age, were randomly sampled. The mothers were interviewed by telephone and complete interviews were conducted with $92 \%$ of them. Of the 795 mothers, $84 \%$ had a relative or friend and $24 \%$ had a physician or nurse within their social networks whom they could contact for advice and support when their children became ill. No associations were found between the mothers' access to advice and support from medical professionals within their social networks and the number of formal medical contacts for their young children's illnesses. Similarly, no associations were found between the mothers' access to advice and support from lay persons in their social networks and the use of medical services for their children.
\end{abstract}

\section{INTRODUCTION}

Previous research into the effects of social networks on children's use of medical services has been contradictory. In an early key paper Kinley (6) studied consultation behaviour of working class families from Aberdeen, Scotland. He found notable differences between social networks of utilizers and underutilizers of antenatal obstetric care. When the babies of these families became ill, a much higher proportion of the underutilizers got advice from a relative or friend as compared with the utilizers. In a British study by Scambler et al. (10) large kinship networks appeared to predispose women to consult their general practitioner while large friendship networks seemed to reduce service use. In a study from the United States which included 513 children under five years of age enrolled in a prepaid group practice, Horwitz et al. (4) found social networks and health attitudes to be important predictors of acute paediatric care utilization. Individuals with large non-dispersed networks and a tendency to use their networks were more likely to use paediatric health services than those without these 
characteristics. The authors described an interaction between the propensity to seek care and a tendency to use network members. Among families showing low use of social networks, the propensity to seek care was positively associated with use of medical services. No such association was found among the families with high network use.

It is important to take social class into account when studying relationships between social networks and utilization of medical services. In a general practice study of consultation patterns for children under 12 years of age, conducted in Scotland, Campion and Gabriel (2) reported economically disadvantaged and less educated families to be high consumers of medical services more often than families without these social characteristics. In a recent general practice study from Glasgow (7) single mothers with low educational attainment and receiving income support were overrepresented among the mothers of children under 10 years of age seen frequently out of hours when compared with a control group of mothers from the same practice. No relationship was found between the presence of a close relative living nearby and the children's use of medical care.

In Sweden, as in most other developed countries, the proportion of the adult population working within health and medical services has increased steadily throughout the last decades. In 1970, $13 \%$ of gainfully employed Swedish women and $1 \%$ of men worked within health and medical care according to Statistics Sweden (11). In 1985, the corresponding figures were $25 \%$ for women and $3 \%$ for men according to Statistics Sweden (12). During the last 15 years, the number of physicians and nurses in Sweden has more than doubled. It is obvious that an increasing number of families with young children have access to support and medical advice from medical professionals from within their own social networks. Consequently, the aim of this study was to examine if medical professionals within the social networks of other families have any impact on the use of formal medical care for young children's minor illnesses.

\section{MATERIAL AND METHODS}

\section{The study setting}

The study area is the county of Uppsala, Sweden, which has 255,000 residents. In $1986,3,225$ children were born in the county. The city of Uppsala, with a population of 158,000 , is located in the central part of the county and lies $70 \mathrm{~km}$ (44 miles) north of Stockholm. District nurses and district physicians employed by the county council are responsible for major elements of primary health care, including medical services for young children. Most specialized outpatient care and nearly all inpatient care is 
provided by the large University Hospital in Uppsala. The greater part of the costs for medical services is paid through the National Social Insurance system. All visits to district nurses are free of charge, while a fee is charged for physician visits.

\section{Two samples of mothers}

Two groups of mothers were originally sampled at random to evaluate the effects of a self-care educational programme as described by Rasmussen (8). These groups were sampled in the autumn of 1986 and the spring of 1987 from the Uppsala County Population Register, which contains data about all residents of the county. Mothers who were Swedish citizens, residents of Uppsala County, and whose first child would be 6-12 months of age at the midpoint of planned interview periods were eligible to the study. Sample one comprised 288 mothers whose children were born between October 1, 1985 and March 31, 1986. Sample two comprised 572 mothers whose children were born between April 1, 1986 and September 30, 1986. In total 860 mothers were sampled for this study.

\section{Interview with mothers}

Professional interviewers from Sweden's Central Bureau of Statistics collected the data by telephone. The mean time needed for each interview was 20 minutes. All questions used in this study were exactly the same in 1986 and 1987.

The mothers were asked if their children experienced any episode of symptoms of illness shown in Table 1 . The following questions were asked concerning utilization of medical services: Have you or your husband on behalf of your young child: 1) Contacted a district nurse on duty because of symptoms/illness during the previous three months? Yes/No. If yes, how many times? 2) Contacted a physician because of symptoms/illness during the previous three months? Yes/No. If yes, how many times? The mothers' propensity to seek medical attention for their young children's minor illnesses was measured by an index based on their answers to seven health problem vignettes presented during the interview as described by Rasmussen (8). The vignettes included the following health problems: acute otitis media, upper respiratory tract infection, pneumonia, acute gastroenteritis, high fever, infantile eczema, and wasp stings. After each vignette the mothers were asked if they would seek medical attention. Those mothers who would use formal medical care scored 1 and the other mothers scored 0 . After summation the total score ranged from 0 to 7 .

In addition to questions about family structure, use of child day-care, and the mother's education and occupation, the following questions were asked about the mother's social network: 1) Do you have a relative or a friend outside your household whom you can contact and get advice from if your child becomes ill? Yes/No. If yes, 
who is he/she? 2) What occupation/education does he/she have?

\section{Statistics}

The information collected from sample one in September and October 1986 referred to the summer months June, July and August and September in early autumn. The information collected from sample two in April and May 1987 referred to the winter months January, February, March and April. No information was available from the remaining months of the year. Therefore, no attempt was made to calculate the mean for the number of medical care contacts representative for a complete calendar year. The patterns of utilization are of greater interest than the exact levels. Therefore, the use of medical services was calculated as an unweighed mean for all the interviewed mothers.

The data were analysed using the SAS programme package (1989). Bivariate analysis was made by Student's t-test. Multiple linear regression analysis was performed using $\log$ transformation of the number of contacts to medical services to satisfy model assumptions.

\section{RESULTS}

\section{Non-response}

A complete interview was made with $795(92 \%)$ of the 860 mothers. The mean age of their children was 10 months at the time of the interviews. Comparisons between respondents and non-respondents showed no significant differences with regard to age and marital status of the mothers or the proportion of families living in rural areas. Only three mothers refused to be interviewed. All other non-respondents could not be reached through several (at least two) telephone calls.

\section{Illnesses, propensity to use medical care and use of services}

More than $90 \%$ of the young children had experienced at least one episode of symptoms of illness during the three months period (Table 1). For more than half of the children $(57.5 \%)$, a face-to-face visit or a phone call had been made to a district nurse or a physician because of symptoms/illness (Table 2). Among the children, 32 (4\%) had a chronic disease or a handicap, according to their mothers. A district nurse or a physician had been seen significantly more often by the children who had had four or more episodes of symptoms of illness than among those with $0-1$ such episodes ( $\mathrm{P}<0.001)$ (Table 3). Equivalent comparisons for use of medical care between the children who had 2-3 episodes of symptoms and those with fewer and/or more episodes showed no statistically significant differences. 
Table 1. Illness symptoms among 795 children 6-15 months of age as perceived by their mothers during the previous three months.

Type of symptom with symptoms

Percentage of children

Runny Nose

Temperature above 38 degrees Celsius

Diarrhoea

Coughing repeatedly

Vomiting repeatedly

Eczema

Breathing difficulties

None of the symptoms above

Five or more of the symptoms above
81.9

56.5

29.4

25.9

16.0

13.2

9.6

8.7

8.0

Table 2. Contacts with a district nurse or a physician for 795 children 6-15 months of age during three months.

Type of contact

Percentage of children with a contact
Mean number of

contacts per child

Any type of medical contact due to illness

District nurse 57.5 1.08

Physician due to any illness 36.4 0.38

Physician visit for acute otitis media collected 
Table 3. Use of medical care during three months for health problems among children 6-15 months of age according to occurrence of illness symptoms and the mothers' propensity to seek medical attention.

Independent variable Number of children Number of contacts with a district nurse or a physician due to perceived health problem of the child

Number of episodes with illness symptoms the previous 3 months

$\begin{array}{lll}0-1 & 252 & 0.41 \\ 2-3 & 384 & 1.08 \\ 4 \text { or more } & 159 & 1.74\end{array}$

Mother's propensity to

seek medical care

Low

412

1.05

High

383

\section{Socio-demographic factors}

At the time of the interview the mean age of the 795 mothers was 27.5 years (range 16 to 44 years). Of the mothers, $45 \%$ had at least three years of upper secondary school education (high school diploma), and $55 \%$ had lower education (no high school diploma). Medical care had been used significantly more often among the children whose mothers were under 25 years of age compared with those whose mothers were 35 years of age or above $(P<0.01)$ (Table 4). The children whose mothers were 25-34 years of age did not differ from the other two groups with respect to medical care utilization. No significant associations were found between family type and the mother's education on the one hand, and the number of contacts with medical services on the other hand.

Between the children whose mothers were employed in child day-care services and those employed within the medical field, no significant difference was found with respect to the number of contacts with a nurse or physician because of perceived health problems $(\mathrm{P}=0.596)$ (Table 4$)$. At the time of this investigation, a majority of 
the mothers was at home in order to take care of their young children. Only $6 \%$ of all 795 children were enrolled in paid day-care outside their own home.

Table 4. Use of medical care during three months for health problems among children 6-15 months of age according to socio-demographic factors and social networks.

Independent variable

Number of children
Number of contacts with a district nurse or a physician due to perceived health problem of the child

\section{Age of mother}

-24 years

221

1.20

25-34 years

502

1.07

35- years

71

0.80

Family type

Single mother

47

1.13

Two parents

742

1.08

Mother's education

High school diploma

413

1.11

No high school diploma

334

1.03

Mother's occupation

Medical profession*

308

1.00

Child day-care

95

1.32

Other occupations

388

1.08

Access to advice from social network when the child is ill

No

Yes, by a lay person

Yes, by a physician or

a nurse
123

479

190
1.12

1.00

1.25

*) Including pharmacy

\section{Social networks}

When the young children were ill, 669 mothers $(84 \%)$ had a relative or friend who could give support and advice. Of these 669 mothers, $40 \%$ would, in the first in- 
stance, seek advice from a friend, $38 \%$ from their own mother, $14 \%$ from one of their own siblings, $5 \%$ from their mother-in-law, $2 \%$ from their father, and $1 \%$ would seek advice from their father-in-law. Of the 669 persons giving support and advice concerning the child's health problem, $14 \%$ were physicians, $15 \%$ were nurses, $18 \%$ belonged to other groups of medical care personnel, $14 \%$ were employed in municipal child day-care services, and $39 \%$ belonged to other professions. Of all 795 mothers, $24 \%$ had a nurse or a physician in their social network from whom they could get advice and support if their young child became ill.

No significant difference was found with respect to use of medical care for young children's illnesses between mothers who had access to advice and support from a nurse or a physician in their social networks and those who had only access to lay advice. No significant difference was present between the mothers who had access to lay advice and those who had no access to advice and support in their social networks. Lastly, no difference was found between the mothers who had a medical professional in their social network and those who had no access to advice and support (Table 4). Among the mothers who had someone to contact for advice and support about their young children's health problem, no significant differences with respect to use of medical care were found between those who in the first instance would seek advice from a friend and those who would rely on family relationships.

All comparisons shown in Table 4 were repeated after excluding the 32 children who had a handicap or a chronic disease. This change affected results only marginally. Multiple linear regression analysis was performed using the logarithm of the number of medical contacts as the outcome variable (Table 5). The children's number of illness symtoms and their mothers' propensity to seek medical attention were significant predictors of medical care utilization. However, the social networks and socio-demographic factors shown in Table 4 were not significant predictors. The association between the mother's age and use of medical care, found in the bivariate analyses, disappeared in the regression analysis. No interaction was detected between the mother's propensity to seek medical care and social network variables with regard to use of medical care.

\section{DISCUSSION}

In Sweden, working mothers have the legal right to remain at home during the first 12 months after childbirth, with $90 \%$ salary reimbursement. The fact that the mothers were often at home when called by interviewers contributed to the very low nonresponse ratio $(8 \%)$. The mothers were originally sampled for a study of the effects of an educational programme in self-care for young children's health problems as 
described by Rasmussen (8). The outcome variable of that study was the mothers' knowlewdge about self-care. No association was found between the mothers' participation in this programme and use of medical services for their children's minor illnesses. Therefore, one could disregard this programme as a confounding factor in the present context. The data were not specifically collected for answering the present study questions. This explains why information about social network was collected by short and clear-cut questions rather than by any of the social network scales that have been described previously $(1,14)$. The focus of the present study is on medical professionals in social networks rather than the importance of the various dimensions of social networks scales (size, frequency of contacts, integration, strength of ties, etc.). None of the validated network scales would have been instrumental in explaining the importance of medical professionals in social networks as regards use of medical care.

Table 5. Multiple linear regression analysis with the natural logarithm of the young children's number of medical care contacts due to illness as the dependent variable.

\begin{tabular}{llll}
\hline $\begin{array}{l}\text { Independent } \\
\text { variable }\end{array}$ & $\begin{array}{l}\text { Beta- } \\
\text { estimate }\end{array}$ & $\begin{array}{l}95 \% \text { confidence } \\
\text { interval for beta- } \\
\text { estimate }\end{array}$ & P-value \\
\hline $\begin{array}{l}\text { Intercept } \\
\text { Child 's number of epi- } \\
\text { sodes of symptoms of ill- } \\
\text { ness during three months } \\
\quad 0-1 * \\
\begin{array}{l}2-3 \\
4 \text { or more }\end{array}\end{array}$ & 0.02 & $(-0.11 ; 0.16)$ & 0.71 \\
& & & \\
$\begin{array}{l}\text { Mother's proneness to } \\
\text { seek medical attention }\end{array}$ & 0.37 & $(0.29 ; 0.45)$ & 0.0001 \\
\end{tabular}

*) Reference category

It is a limitation that use of medical care was measured rather crudely in this study. It is possible, but not very likely, that relationships between social networks and use of medical services would have been found if more detailed information had been available about services use. That no association was found between socio-demographic factors and use of medical care is not astonishing for a Scandinavian country. From this point of view, the results of the present study are in accordance with the findings 
of a recent Swedish diary study (5).

It is interesting that as many as $24 \%$ of this non-selected group of Swedish mothers had a physician or nurse in their social network who could give support and advice. In 1985, the proportion of the adult population working within health and medical services was $15 \%$ in Uppsala County and $14 \%$ for Sweden as a whole, according to Statistics Sweden $(11,12)$. However, authorized physicians comprised $0.8 \%$ of the adult working population of Uppsala County and $0.5 \%$ for the entire country. In $1990,42 \%$ of the population in Uppsala County between the ages of 25 and 34 years had at least three years of upper secondary school education, compared with $34 \%$ of the total population of Sweden in the same age range according to Statistics Sweden (1990). Young families with a high socio-economic status and higher education are thus more common in Uppsala County than in Sweden as a whole. Such families are more likely to have physicians in their social network than young families with low socio-economic status. Therefore, Uppsala County may not be representative for the entire country regarding the availability of medical professionals in the social networks of young families.

This study shows that $40 \%$ of the mothers sought advice and support from a friend and that a slightly lower proportion sought advice from their own mothers. In a Scottish study by Duncan et al. (3), as well as in McKinlay's study (6), the parent's own mother was the most common source of lay advice. However, in the study by Duncan et al. (3), friends were ranked as the main source of advice only by $5 \%$.

That access to lay advice in social networks was not associated with use of medical services for young children's minor illnesses is consistent with the findings of the Swedish diary study by Höjer et al. (5). However, the results of the present study are not in accordance with the study by Horwits et al. (4), which showed that large nondispersed networks may increase use of medical services. It is also notable that the interaction between the propensity to seek medical attention and the use of social networks described by Horwits et al. (4) could not be reproduced in the present study. Differences of measurements, medical care systems, and beliefs about health and disease may explain the differences uncovered between Sweden and the United States.

In this study, no difference was found with respect to medical care utilization for young children's illnesses between mothers who would rely on kinship networks and those who would primarily rely on friendship networks. The difference between these types of networks proposed by Scambler et al. (10) could thus not be confirmed. When interpreting this discrepancy, the large differences in the nature of the information sources should be kept in mind. Medical professionals in social networks may 
affect mothers' perceptions of their children's symptoms in various ways. In some social networks, pro-medical health beliefs may be transferred to the mothers and thereby increase medical care utilization for young children's minor illnesses. In other social networks, advice and support and sometimes drug prescription to ailing young children may satisfy the needs of medical care and thereby reduce utilization of formal medical services. In the present study, these forces may have cancelled out each other.

\section{REFERENCES}

1. Bowling, A.: Social Support and Social Networks: Their Relationship to the Successful and Unsuccessful Survival of Elderly People in the Community. An analysis of concepts and a review of the evidence. Family Practice 8: 68-83, 1991.

2. Campion, P.D. \& Gabriel, J.: Child Consultation Patterns in General Practice Comparing "High" and "Low" Consulting Families. Br Med J 288: 1426-8, 1984.

3. Duncan, J.K., Taylor, R.J. \& Fordyce, I.D.: Factors Associated with Variation in the Consultation Rates of Children Aged Under Five Years. J R Coll General Pract 37: 251-4, 1987.

4. Horwitz, S.M., Morgenstern, H. \& Berkman, L.F.: The Impact of Social Stressors and Social Networks on Pediatric Medical Care Use. Medical Care 23: 946-59, 1985 .

5. Höjer, B., Sterky, G. \& Widlund, G.: Acute Illnesses in Young Children and Family Response, Acta Paediatr Scand 76: 624-30,1987.

6. McKinlay, J.B.: Social networks, Lay Consultation and Help Seeking Behaviour. Social Forces 51: 275-92,1973.

7. Morrison, J.M., Gilmour, H. \& Sullivan, F.: Children Seen Frequently Out Of Hours in One General Practice. Br Med J 303: 1111-14,1991.

8. Rasmussen, F.: Mothers' Benefit of a Self-Care Booklet and a Self-Care Educational Session at Child Health Centres. Soc Sci Med 29: 205-12,1989.

9. SAS Institute Inc.: SAS/STAT User's Guide, Version 6, Volumes 1 and 2. Cary, North Carolina: SAS Institute Inc, 1989.

10. Scambler, A., Scambler, G. \& Craig, D.: Kinship and Friendship Networks and Women's Demand for Primary Care. J R Coll General Pract 31: 746-50, 1981. 
11. Statistics Sweden: Population and Housing Census 1970, Part 13. Stockholm, Sweden 96, 1975.

12. Statistics Sweden: Population and Housing Census 1985, Part 7. Stockholm, Sweden 75, 1989.

13. Statistics Sweden: Education of the Population. Stockholm, Sweden, 1990.

14. Östergren, P.O., Hansson B.S., Isacsson, S.O. \& Tejler, L.: Social Network, Social Support and Acute Chest Complaints Among Young and Middle-Aged Patients in an Emergency Department - A Case-Control Study. Soc Sci Med 33: 257-67, 1991.

Offprint request to: Finn Rasmussen

Department of Paediatrics

Children's University Hospital

S-751 85 Uppsala, Sweden 